\title{
FEASIBILITY OF INTRAOPERATIVE BALLOON ANGIOPLASTY AND ADDITIONAL STENT PLACEMENT OF ISOLATED STENOSIS OF THE BRACHIOCEPHALIC TRUNK
}

Alexander Ruebben, MD

Serena Tettoni, MD

Pierluigi Muratore, MD

Denis Rossato, MD

Daniele Savio, MD

Massimo Conforti, MD

Franco Nessi, MD

Claudio Rabbia, MD
Objective: We sought to evaluate the feasibility and results of intraoperative balloon angioplasty and additional stent placement of isolated stenosis of the brachiocephalic trunk. Patients and methods: Between May 1993 and October 1996, we treated eight patients with local stenosis of the innominate artery. Seven lesions were situated in the proximal and one in the middle third of the brachiocephalic trunk. Five patients were men and three were women, with ages ranging from 55 to 72 years (mean 59.5 years). All stenoses provoked severe blood flow reduction and caused clinical symptoms. Procedures were performed in an operating suite with fluoroscopic imaging capabilities. Through an anterolateral cervical approach the right common carotid artery was surgically exposed and then clamped to avoid atheroembolization during the subsequent procedure. Retrograde catheterization was performed to reach the stenosis of the brachiocephalic trunk. The lesion was dilated with a balloon catheter and successively stented. Follow-up examinations (color-coded duplex sonography, accompanied by clinical inspection and systolic blood pressure) were scheduled every 6 months. Results: In all patients the dilation of the stenosis of the innominate artery and the stent placement were successful without any side effects. No embolic events or other complications occurred. The postintervention angiography showed successfully dilated stenoses and patent stents in all cases. The technical success rate was $100 \%$. Conclusions: On the basis of our preliminary data, we believe that, in selected patients, intraoperative balloon angioplasty of stenosis of the innominate artery with stent placement from the right common carotid artery approach is a safe and effective alternative to conventional operations. (J Thorac Cardiovasc Surg 1998; 115:1316-20)
$\Delta$ therosclerotic lesions of the brachiocephalic Atrunk are relatively rare compared with other types of vascular diseases. Only $1.5 \%$ of occlusive extracranial lesions causing cerebrovascular deficits are located in the innominate artery. ${ }^{1}$ Symptoms may be as varied as ischemia of the upper right extremity, vertebrobasilar territory deficits, ocular disturbance, and cortical ischemic events. Several transthoracic or extrathoracic operative

From Radiologia del Pronto Soccorso, Azienda Ospedaliera San Giovanni Battista, Torino, Italy.

Received for publication March 13, 1997; revisions requested Sept. 11, 1997; revisions received Jan. 9, 1997; accepted for publication Jan. 9, 1998.

Address for reprints: Claudio Rabbia, Radiologia del Pronto Soccorso, Azienda Ospedaliera San Giovanni Battista, Corso Bramante 88, 10126 Torino, Italy.

Copyright (C) 1998 by Mosby, Inc.

$0022-5223 / 98 \$ 5.00+0 \quad \mathbf{1 2 / 1 / 8 8 8 2 0}$ procedures have been devised for innominate artery reconstruction. However, median sternotomy with direct endothoracic repair is recommended because of good early and long-term results. ${ }^{2-5}$ Nevertheless, this procedure is not without risks such as hemorrhage, embolism, aortic dissection, infection, or death. ${ }^{5}$

Recent literature shows a mortality and an incidence of neurologic events of $5.4 \%$ and $3.4 \%$, respectively. ${ }^{5}$

As an alternative to reconstructive surgery, percutaneous transluminal angioplasty of the innominate artery has only been mentioned in isolated cases to date..$^{6-17}$ This report therefore describes our experience in intraoperative balloon angioplasty and additional stent placement of isolated stenosis of the brachiocephalic trunk with cerebral protection ensured by common carotid artery clamping. 
Table I. Percentage of stenosis and pressure gradient before intervention

\begin{tabular}{ccccc}
\hline $\begin{array}{c}\text { Patient } \\
\text { No. }\end{array}$ & $\begin{array}{c}\text { Age } \\
(y r)\end{array}$ & Sex & $\begin{array}{c}\text { Percentage } \\
\text { of stenosis }\end{array}$ & $\begin{array}{c}\text { Blood pressure between } \\
\text { the right and left arm }\end{array}$ \\
\hline 1 & 55 & Male & 80 & $25 \mathrm{~mm} \mathrm{Hg}$ \\
2 & 61 & Male & 95 & $40 \mathrm{~mm} \mathrm{Hg}$ \\
3 & 56 & Female & 85 & $30 \mathrm{~mm} \mathrm{Hg}$ \\
4 & 72 & Male & 90 & $35 \mathrm{~mm} \mathrm{Hg}$ \\
5 & 57 & Female & 85 & $30 \mathrm{~mm} \mathrm{Hg}$ \\
6 & 59 & Female & 90 & $35 \mathrm{~mm} \mathrm{Hg}$ \\
7 & 60 & Male & 90 & $30 \mathrm{~mm} \mathrm{Hg}$ \\
8 & 56 & Male & 95 & $35 \mathrm{~mm} \mathrm{Hg}$
\end{tabular}

\section{Patients and methods}

Between May 1993 and October 1996, we treated eight patients with local stenosis of the innominate artery. Seven lesions were situated in the proximal third (two of which were ostial lesions) and one in the middle third of the brachiocephalic trunk. Five patients were men and three were women, with ages ranging from 55 to 72 years (mean age 59.5 years).

The pathogeneses of the stenosis was arteriosclerosis. All patients had a history of smoking (more than 25 cigarettes a day). Five of eight patients had hypertension that necessitated medical treatment. Diabetes mellitus was present in two patients, and one patient had hypercholesterolemia. All stenoses provoked severe blood flow reduction and caused clinical symptoms. Four patients described right arm claudication, three patients had vertebrobasilar insufficiency with ataxia, and one patient had transient right hemispheric ischemic events. Three of eight patients had associated cardiovascular disease: coronary artery disease in two cases and peripheral arterial occlusive disease in one patient.

Before we started the interventional procedures, we evaluated the stenosis of the innominate artery with color-coded duplex sonography and selective arch angiography (Fig. 1). Systolic blood pressure was measured on both arms to determine the gradient before the intervention (Table I). All patients had reduced flow to the right common carotid artery. In five patients (patients 2, 4, 6, 7 , and 8) there was a complete steal of the right vertebral artery and in three (patients 1,3, and 5) an incomplete steal. In patient 2 an additional incomplete steal of the right internal carotid artery was recorded, and in patient 6 an incomplete steal of the right external carotid artery was found. An additional tight stenosis of the right internal carotid artery was present in patient 1 . Furthermore, in three patients (patients 4, 7, and 5) we found an asymptomatic moderate stenosis of the contralateral internal carotid artery.

All procedures were performed in an operating suite with fluoroscopic imaging capabilities (Philips BV 29 Mobile Image System, Best, The Netherlands). All angiograms were printed on a graphic printer (Sony Video UP-910, Tokyo, Japan). Three patients were operated on with general anesthesia, and five had local anesthesia. The procedures were monitored by measuring blood pressure of the right and left arm during and after the procedure.

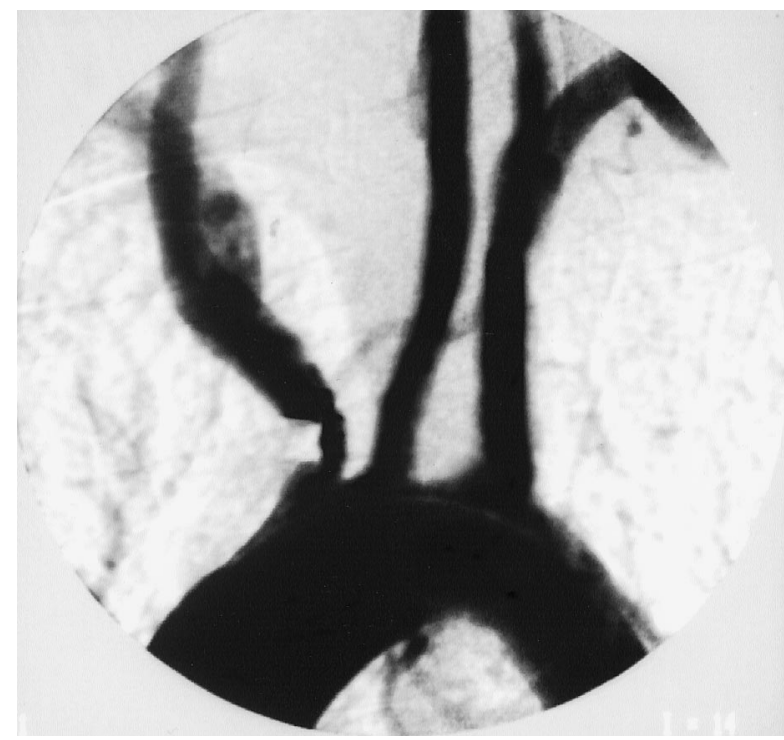

Fig. 1. Proximal stenosis of the brachiocephalic trunk.

Through an anterolateral cervical approach the right common carotid artery was surgically exposed. To avoid atheroembolization during the interventional procedure the artery was clamped. Proximal to the vessel clamp, an $8 \mathrm{~F}$ introducer sheath was inserted into the vessel. Anticoagulation therapy with heparin (5000 IU) was administered intraarterially at the beginning of the procedure. An intraoperative angiogram through the introducer sheath was performed to identify the position of the lesion. The stenosis of the innominate artery was traversed under direct fluoroscopic visualization with a J or straight-tipped hydrophilic guide wire (Terumo Corporation, Tokyo, Japan). The balloon size was chosen after comparing the diameter of the innominate artery measured distal to the stenosis by ultrasound, with the angiogram demonstrating the stenotic lesion. A balloon catheter $(8$ to $10 \mathrm{~mm}$ in diameter) was guided across the stenosis and inflated twice up to the normal width of the vessel at 5 to 10 atmospheres. In all patients a Palmaz stent (P308) (Johnson \& Johnson Interventional System, Warren, N.J.) was placed in the stenotic area and dilated to the same diameter as the balloon catheter to maintain a postangioplasty lumen. In the two patients in whom the stenoses involved the origin of the innominate artery, the stent was placed with its proximal end 2 or $3 \mathrm{~mm}$ into the aortic arch. After the stent had been positioned, a postintervention angiogram through the introducer sheath was performed to assess the degree of luminal enlargement, patency of the vessels, evidence of intimal damage, and to exclude embolization of other branches (Fig. 2). After removal of the introducer sheath, the common carotid artery was flushed to avoid embolization after angioplasty.

The surgically exposed vessel was repaired with a continuous suture of 6-0 polypropylene (Ethicon, Inc., Somerville, N.J.) and in seven of eight patients, the wound was closed in the standard manner. During the same intervention, the incision in patient 1 had to be enlarged 


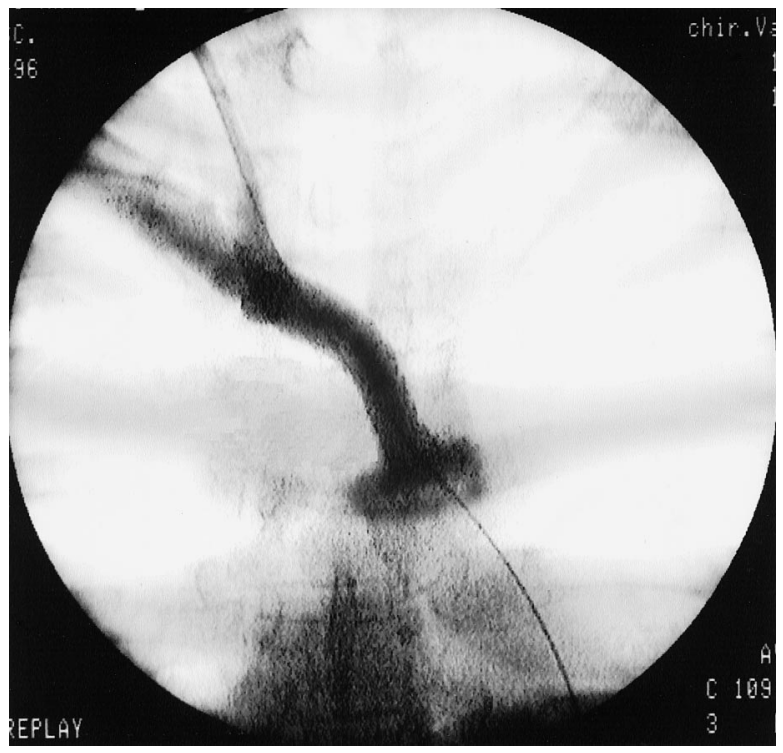

Fig. 2. Intraoperative angiogram after angioplasty and stent placement.

to treat a concomitant ipsilateral internal carotid artery stenosis by endarterectomy. Minimum blood loss, on average $40 \mathrm{ml}$, occurred. Mean common carotid crossclamp time was 28 minutes, and operative time ranged from 45 to 92 minutes (mean 68 minutes). No anticoagulation therapy was administrated after the procedures; however, oral antiplatelet therapy with acetylsalicylic acid (300 mg/day) was implemented. Before the patient was discharged, the systolic blood pressure of both arms was measured, and color-coded duplex sonography was performed.

Follow-up examinations (color-coded duplex sonography accompanied by clinical inspection and systolic blood pressure) were scheduled every 6 months.

\section{Results}

In all patients the dilation of the stenosis of the innominate artery and the stent placement were successful without any side effects. The pressure gradient between the right and left arm resolved immediately. In all patients a postintervention angiogram showed the adequately dilated stenoses and patent stents. The technical success rate was $100 \%$.

No distal embolism with neurologic events, innominate artery dissection, rupture, occlusion, or neck hematoma occurred. No respiratory or cardiac complications were recorded during or after intervention. No patient died during or after intervention. The postoperative course was uneventful in all patients. Examinations performed with color-coded duplex sonography after the procedure confirmed normalization of flow through the right carotid artery and demonstrated antegrade flow in the right vertebral artery.

Preoperative symptoms such as right arm claudication (four patients) and vertebrobasilar insufficiency with ataxia (three patients), disappeared after intervention. The patient who had had transient right hemispheric ischemic events no longer experienced neurologic deficits.

Long-term follow-up with color-coded duplex sonography after a mean time of 17 months (range 1 to 42 months) revealed stents with normal flow patterns and no signs of restenosis. At the latest follow-up the primary patency rate was $100 \%$.

Blood pressure measurements remained stable and no symptoms of restenosis of the brachiocephalic trunk occurred. No complications related to the procedures were experienced during this time.

\section{Discussion}

At present operative revascularization of innominate artery stenosis is recommended for lesions causing symptoms related to hypoperfusion or embolization. ${ }^{4}$ Surgical management remains controversial, but most authors recommend direct transthoracic repair for good operative candidates, either bypassing the occluded vessels or, in selected cases like isolated obstructions or concomitant coronary artery bypass grafting, carrying out endarterectomy. ${ }^{4,5,18}$ Despite good immediate and long-term results with a 10-year patency rate of about $96.3 \%,,^{5}$ these procedures are associated with the risk of major complications. Neurologic events and death can occur in $3.4 \%$ and $5.4 \%$ of cases, respectively. ${ }^{5}$

An extrathoracic approach is primarily used for elderly patients, who are poorly suited to tolerate a sternotomy, or for patients with a severely calcified ascending aorta. ${ }^{4}$ A variety of procedures have been developed to avoid sternotomy. The most common extrathoracic reconstruction for innominate artery stenosis is the axilloaxillary bypass. In fact, the axillary artery as donor vessel is convenient because of its superficial location and the low risk of phrenic nerve injury. ${ }^{18}$ This procedure has less morbidity (incidence of perioperative myocardial infarction, stroke, and death $<2 \%$ ), but long-term patency is often reduced. The estimated patency varies from a 3 -year rate of about $76 \%$ to a 5-year rate of $90 \% .{ }^{19-21}$ Unfavorable graft orientation, subcutaneous transsternal position, and extent of disease, especially in the inflow vessels, ${ }^{4}$ are risk factors for late graft occlusion.

Alternatively, balloon angioplasty of the su- 
praaortic vessels has been suggested, with a good technical success and a low incidence of major and minor complications of up to $1.5 \%$ and up to $2.02 \%$, respectively. ${ }^{8,12-16}$ However, most of these reports described cases of stenotic lesions of the subclavian artery and very few involved the innominate artery. Although the dilation of a stenosis of the subclavian artery may eventually compromise the vertebral artery-which is, however, often protected by a retrograde flow $^{22}$ - the dilation of an innominate artery stenosis risks embolization into the nonprotected carotid artery. Therefore brachiocephalic trunk lesions have been reluctantly treated exclusively with balloon angioplasty and are considered at relatively high risk for thromboembolic events. Nevertheless, no study in the literature quantifies the risk rate for angioplasty of the innominate artery.

Techniques proposed for cerebral protection to minimize the possible risk of embolization include manual compression of the distal cephalic branch, use of an independent occlusion balloon or intervention with interrupted flow by vessel loop or clamp and, successively, "washout" through an arteriotomy. ${ }^{7,9-11}$ In all studies in the literature no case of atheroembolization occurred, ${ }^{7,9-11}$ when balloon dilation of innominate artery stenoses combined with cerebral protection was performed.

Despite the possibility of measuring the pressure gradient across the stenosis during the procedure, we believed that the presence of the clamp could falsify the gradient, and therefore preferred the blood pressure measurement of both arms to monitor the gradient before and after angioplasty.

Special anesthesiologic techniques are required in the case of stenotic or obstructive lesions of the contralateral artery (extracranial and intracranial vessels), which may compromise the compensatory flow because of the reduced flow in the innominate artery. In fact, when the contralateral side was involved by the atheromatous process, the procedures were performed with the patient under general anesthesia. Three such cases were in our study. High doses of barbiturates and neuroleptic agents are administered to reduce brain metabolism as a protection against ischemic events. A shunt is not necessary for these operations because of the external carotid-to-internal carotid artery collateralization and the brief carotid artery crossclamp time.

On the other hand, when stenotic lesions were only ipsilateral, the procedures were performed with the patient under local anesthesia. Communication with the patient during the procedures enables the operator to detect any initial disturbance of consciousness resulting from insufficient collateral arteries.

With common carotid clamping and postprocedural flush-out no embolization occurred. Nevertheless, we are aware of the persistence of a minimal embolic risk during the dilation or injection of contrast material, such as embolization into a vertebral artery with antegrade flow or into the aortic arch reaching contralateral supraaortic vessels. We believe there is increased risk of embolization if the left common carotid artery is involved or closely approximates the origin of the innominate artery. Therefore, in such a case, we recommend a reconstructive operation.

After complete dilation of the stenosis, an additional stent was placed in the lesion: first, to maintain the achieved lumen of the vessel; second, to smooth the atheromatous lesion to avoid turbulent flow that may induce microembolism and to reduce the risk of restenosis. Lesions of the innominate artery have restenosed when no stent has been used. ${ }^{6,9,14}$

Queral and Criado ${ }^{17}$ treated brachiocephalic lesions by introducing the prosthesis after a preliminary balloon dilation of only $4 \mathrm{~mm}$. The results of this study demonstrate the feasibility of treating innominate artery stenosis with angioplasty and a Palmaz stent. However, in our opinion, it is important that the stenosis is dilated to the maximum width of the vessel before placing the stent to test the distensibility of the artery and therefore the feasibility of stent placement.

To prevent a possible restenosis in ostial lesions the stent was placed 2 or $3 \mathrm{~mm}$ into the aortic arch to completely cover the atheromatous plaque. No complications related to the protrusion were registered during the follow-up.

This new technique offers a safe and effective approach to stenoses of innominate arteries because it is less invasive than conventional transthoracic or extrathoracic surgery and offers excellent early and midterm results. This even allows the treatment of elderly patients with a high risk of thoracotomy and patients who have previously undergone coronary artery bypass grafting. Patients who cannot tolerate general anesthesia may also be treated because in most cases local anesthesia is used.

Finally, the cost of intraoperative balloon angioplasty is lower than a traditional operative procedure, owing to the reduced hospitalization period.

On the basis of our preliminary data, we believe 
that, in selected patients and considering the anatomy of the supraaortic vessels, angioplasty of the innominate artery with stent placement during right common carotid artery exposure and clamping is a safe and effective alternative to conventional surgery, although the need for long-term follow-up data is required.

\section{REFERENCES}

1. Damme HV, Caudran D, Defraigne JO, Limet R. Brachiocephalic arterial reconstruction. Acta Chir Belg 1992;92:3745 .

2. Crawford ES, Stowe CXI, Pewers RW. Occlusion of the innominate, common carotid and subclavian arteries: longterm results of surgical treatment. Surgery 1983;94:781-91.

3. Cormier F, Ward A, Cormier JM, Laurian C. Long-term results of aorto-innominate and aorto-carotid PTFE bypass grafting for atherosclerotic lesions. J Vasc Surg 1989;10:13542.

4. Reul GJ, Jacobs MJHM, Gregoric ID, et al. Innominate artery occlusive diseases: surgical approach and long-term results. J Vasc Surg 1991;14:405-12.

5. Kieffer E, Sabatier J, Koskas F, Bahnini A. Atherosclerotic innominate artery occlusive disease: early and long-term results of surgical reconstruction. J Vasc Surg 1995;21:32637.

6. Theron J, Courthereoux P, Henriet JP, Pelouze G, Derlon JM, Maiza D. Angioplasty of supraaortic arteries. J Neuroradiol 1984;11:187-200.

7. Vitek JJ, Keller FS, Duvall ER, Gupta KL, Chandra-Sekar B. Brachiocephalic artery dilation by percutaneous transluminal angioplasty. Radiology 1986;158:779-85.

8. Higashida RT, Hieshima GB, Halbach VV. Advances in the treatment of complex cerebrovascular disorders by interventional neurovascular techniques. Circulation 1991;83(Suppl): I196-206.

9. Gobin Y, Hassani R, Batellier J, Casasco A, Aymard A, Merland JJ. Transluminal angioplasty of the brachiocephalic artery with cerebral protection. J Mal Vasc 1991;16:188-90.

10. Lowman Bg, Queral LA, Holbrook WA, Estes JT, Bayly B.
The treatment of innominate artery stenosis by intraoperative transluminal angioplasty. Surgery 1981;89:565-8.

11. Derauf BJ, Erickson DL, Castaneda-Zuniga WR, Cardella JF, Amplatz K. "Washout" technique for brachiocephalic angioplasty. AJR Am J Roentgenol 1986;146:849-51.

12. Kachel R, Endert G, Basche S, Grossmann K, Glaser FH. Percutaneous transluminal angioplasty (dilatation) of carotid, vertebral, and innominate artery stenoses. Cardiovasc Intervent Radiol 1987;10:142-6.

13. Dorros G, Lewin RF, Jamnadas P, Mathiak LM. Peripheral transluminal angioplasty of the subclavian and innominate arteries utilizing the brachial approach: acute outcome and follow-up. Cathet Cardiovasc Diagn 1990;19:71-6.

14. Jaschke W, Menges HW, Ockert D, Huck K, Georgi M. PTA of the subclavian and innominate artery: short- and long-term results. Ann Radiol 1989;32:29-33.

15. Selby JB, Matsumoto AH, Tegtmeyer CJ, Hartwell GD, Tribble CG, Daniel TM, et al. Balloon angioplasty above the aortic arch: immediate and long-term results. AJR Am J Roentgenol 1993;160:631-5.

16. Kachel R, Basche S, Heerklotz I, Grossmann K, Endler S. Percutaneous transluminal angioplasty (PTA) of supra-aortic arteries especially the internal carotid artery. Neuroradiology 1991;33:191-4.

17. Queral LA, Criado FJ. The treatment of focal aortic arch branch lesions with Palmaz stent. J Vasc Surg 1996;23:368-75.

18. Owens LV, Tinsley EA Jr, Criado E, Burnham SJ, Keagy BA. Extrathoracic reconstruction of arterial occlusive disease involving the supraaortic trunks. J Vasc Surg 1995;22:217-22.

19. Bentley FR, Hollier LH, Batson RC. Axilloaxillary by-pass for subclavian and innominate artery revascularization. Am Surg 1982;48:70-4.

20. Vogt DP, Hertzer NR, O'Hara PJ, Beven EG. Brachiocephalic arterial reconstruction. Ann Surg 1982;196:541-52.

21. Meyers WO, Lawton BR, Jefferson FR. Axillo-axillary bypass for subclavian steal syndrome. Arch Surg 1979;114: 394-7.

22. Ringelstein EB, Zeumer H. Delayed reversal of vertebral artery blood flow following percutaneous transluminal angioplasty for subclavian steal syndrome. Neuroradiology 1984; 26:189-98.

\section{Availability of Journal back issues}

As a service to our subscribers, copies of back issues of The Journal of Thoracic and Cardiovascular Surgery for the preceding 5 years are maintained and are available for purchase from Mosby at a cost of $\$ 16.00$ per issue until inventory is depleted. The following quantity discounts are available: $25 \%$ off on quantities of 12 to 23 , and one third off on quantities of 24 or more. Please write to Mosby, Inc., Subscription Services, 11830 Westline Industrial Drive, St. Louis MO 63146-3318, or call 800-453-4351 or 314-453-4351 for information on availability of particular issues. If unavailable from the publisher, photocopies of complete issues may be purchased from UMI, 300 N. Zeeb Rd., Ann Arbor, MI 48106, 313-761-4700. 